\title{
A funcionalidade e o desempenho do Portal de Periódicos da CAPES entre pesquisadores das áreas de Comunicação e Ciência da Informação da Universidade Federal da Bahia ${ }^{1}$
}

Rodrigo França Meirelles

\author{
Bibliotecário Documentalista. Consultor em \\ Tecnologia da Informação
}

Raymundo das Neves Machado

Docente do Departamento de Fundamentos e Processos Informacionais/UFBA

A pesquisa busca definir parâmetros e critérios que permitam estudar e aferir a funcionalidade $e \quad o$ desempenho de recursos eletrônicos, no que diz respeito à sua interface com os usuários. Investiga um grupo selecionado de pesquisadores das áreas de Comunicação e Ciência da Informação da Universidade Federal da Bahia. Os resultados da pesquisa indicam que o Portal de Periódicos da Capes apresentou um bom desempenho de acordo com os usuários, mas que necessita de melhorias imprescindíveis em sua usabilidade e legibilidade.

Palavras-chave: Recursos eletrônicos; Usabilidade; Legibilidade.

\section{The functionality and performance of CAPES Journal Gateway among researchers of the Communication and Information Science areas at the Federal University of Bahia}

Extrato da Monografia apresentada por Rodrigo França Meirelles (MEIRELLES, 2005) na disciplina Estágio Supervisionado do Departamento de Fundamentos e Processos Informacionais do Instituto de Ciência da Informação da Universidade Federal da Bahia. 
The research attempts to define parameters and criteria that allow to study and survey the functionality and the performance of electronic resources regarding their interface with users. A selected group of researchers of the areas of Communication and Information Science of the Federal University of Bahia was investigated. According to the results CAPES Journal Gateway has a good performance, although it needs some essential improvements in the layout and also in its usability and readability.

Keywords: Electronic resources; Usability; Legibility.

Recebido em 10.04.2007 Aceito em 01.10.2007

\section{Introdução}

O Portal de Periódicos da Capes, lançado em 2000, proporciona à comunidade científica brasileira o acesso à versão eletrônica dos principais veículos de comunicação da ciência, ou seja, dos periódicos científicos, como também a bases de dados internacionais, permitindo desse modo uma infra-estrutura moderna e democrática de acesso e posse da informação. Embora represente um recurso hoje indispensável à produção científica e tecnológica nacional, o Portal tem sido pouco estudado, sobretudo nos aspectos que dizem respeito à sua aceitação e efetivo uso pela comunidade científica.

Nesse contexto, o estudo teve como objetivo investigar um grupo selecionado de pesquisadores das áreas de Comunicação e Ciência da Informação, pertencente ao quadro docente efetivo da Universidade Federal da Bahia (UFBA), para verificar a aceitação e satisfação que eles demonstram em relação à funcionalidade e ao desempenho do Portal de Periódicos da CAPES. Para atingir este objetivo decidiu-se privilegiar o diagnóstico de sua interface eletrônica, analisando o layout e suas características mais significativas, com a finalidade de definir critérios mínimos de funcionalidade que pudessem ser utilizados para aferir a satisfação do público com os recursos oferecidos pelo Portal.

\section{Portal de Periódicos da Capes}

O periódico científico constitui o principal veículo de transferência da informação científica e tecnológica, sendo uma relevante fonte de informação para a pesquisa. Proporcionar o acesso a esse manancial de informações é colocar a comunidade de pesquisadores atualizada nos avanços de suas respectivas áreas de atuação. Essa foi e continua sendo uma preocupação da Coordenação de Aperfeiçoamento de Pessoal de 
Nível Superior (Capes), que tem um relevante papel na consolidação da pós-graduação stricto sensu em todo o território nacional.

Uma das preocupações da Capes, em sua trajetória de atuação, tem sido a atualização dos acervos de periódicos das Instituições de Ensino Superior (IES) do Brasil. Assim, no período de 1987 a 1991, a CAPES instituiu o Programa de Aquisição Planificada de Periódicos (PAP) para bibliotecas universitárias, com a finalidade de proporcionar a essas instituições a renovação de assinaturas de publicações periódicas. Em 1991 o PAP foi suspenso, ficando sob a égide das instituições mantenedoras das bibliotecas universitárias a renovação das assinaturas das publicações já existentes. Sem dúvida nenhuma isso foi um colapso para as coleções, pois as instituições ficaram impossibilitadas de completá-las, uma vez que o orçamento é cada vez mais reduzido para a aquisição de itens informacionais.

Diante de tal quadro e de grandes prejuízos ao acesso à informação, em 1995 a Capes criou o Programa de Apoio à Aquisição de Periódicos (PAAP), atualizando assim as coleções das bibliotecas das instituições com pós-graduação stricto senso. Vale ressaltar que a manutenção das assinaturas dos periódicos internacionais para várias instituições de ensino no Brasil requer um orçamento de grande porte, não se tratando apenas da assinatura de um único título e sim de vários, para " $n$ " unidades de informação, cobrindo assim todo o País.

Apesar dos escassos recursos orçamentários destinados às bibliotecas universitárias e diante do novo cenário tecnológico, isto é, do avanço das publicações eletrônicas, o Programa de Biblioteca Eletrônica (ProBE) oferecia aproximadamente 841 títulos de periódicos eletrônicos (publicados pela Elsevier Science, Academic Press e High Wire Press) - 0 que representou um ganho no acesso e posse de informações de títulos internacionais de primeira mão a professores, alunos de graduação e pósgraduação de instituições como: Fapesp, USP, Unesp, Unicamp, UFSCar, Unifesp, Bireme e ITA.

Seguindo o exemplo bem sucedido do ProBE, a Capes trilhou o mesmo caminho e lançou em 2000 o Portal de Periódicos da Capes. Iniciativa pioneira no Brasil, o Portal disponibiliza para Instituições Federais de Ensino Superior (IFES), estaduais com programas de pósgraduação, unidades federais de pesquisa com programas com pósgraduação e instituições particulares de excelência (com programas de doutorado de nota maior ou igual a cinco), acesso compartilhado a títulos de periódicos e a bases de dados, incluindo a atualização dos itens contidos no Portal em tempo real, possibilitando pesquisas retrospectivas às coleções, e se constituindo numa grande fonte de pesquisa on line para a comunidade cientifica brasileira, que o recebeu com grande euforia. 


\section{Terminologia utilizada na avaliação do Portal de Periódicos da Capes}

O surgimento da Internet comercial e sua popularização em 1996, com a World Wide Web, impulsionou o crescimento do fluxo de circulação da informação de forma exorbitante e desordenada. As informações passaram a ser lançadas na rede sem maiores preocupações com a sua organização, o que ocasionou obstáculos à sua recuperação, em função do crescimento contínuo e veloz da informação na Web.

O constante crescimento do número de páginas publicadas on-line criou a necessidade de tentar localizar as informações disponibilizadas na $W e b$, impulsionando a criação de metodologias para a organização de sites, blogs e portifolios, criados através de sistemas específicos; bem como a criação de mecanismos de busca como uma tentativa de recuperação da informação disponível com mais velocidade e eficiência. Contudo, com o desenvolvimento de recursos específicos para a Ciência e a Tecnologia (C\&T) e à medida que os pesquisadores dispunham de novos meios de acesso às fontes especializadas de suas áreas, a exigência da comunidade científica por informações precisas e atuais cresceu ainda mais. Ajustados a esse novo contexto, foram criados ambientes que ofereciam instrumentos diversificados como catálogos, bases de dados, periódicos eletrônicos, portais e bibliotecas digitais.

No Brasil, surgiram iniciativas pioneiras de serviços de informação eletrônica em C\&T como, por exemplo, em 1995, o Programa de Informação para Gestão de Ciência, Tecnologia e Inovação (PROSSIGA), incorporado ao IBICT no segundo semestre de 2003, com o intuito de criar serviços de informação e estimular o uso de veículos eletrônicos de comunicação pelas comunidades dessas áreas.

Saracevic e Covi (2000) ressaltam que, embora muitos esforços tenham sido despendidos em pesquisas sobre os recursos eletrônicos, a avaliação desses recursos está ausente na maioria dos trabalhos publicados. A ausência de avaliações precisas e eficientes, que pudessem definir estratégias para a avaliação do Portal de Periódicos da Capes, nos levou a propor uma definição para os termos que caracterizam melhor a avaliação de desempenho do Portal. Foram utilizadas fontes das áreas de informática e design gráfico, onde a legibilidade possui ampla penetração.

Na ABNT, a usabilidade é definida como a "medida na qual um produto pode ser usado por usuários específicos para alcançar objetivos 'específicos' com eficácia, eficiência e satisfação em contextos específicos de uso" (ASSOCIAÇÃO..., 2002, p. 3), afirmando-se que, para aferi-la corretamente, é necessário compreender que o desempenho e a satisfação dos usuários em relação a determinado produto depende da complexidade das interações que se estabelecem entre: (a) o usuário; (b) os objetivos pretendidos de cada produto; $\mathrm{e}$ (c) as características e elementos do contexto de uso.

Levando em consideração que a usabilidade depende não apenas das características do próprio produto, mas também de seu contexto de 
uso, um mesmo produto poderá ter diferentes níveis de usabilidade, dependendo do contexto. Para que se possa medir e especificar a usabilidade de forma mais apropriada são necessárias as seguintes informações:

[...] - Uma descrição dos objetivos pretendidos;

- Uma descrição dos componentes do contexto de uso, incluindo usuários, tarefas, equipamento e ambientes. Esta pode ser uma descrição de um contexto existente ou uma especificação dos contextos pretendidos. Os aspectos relevantes do contexto e o nível de detalhes requeridos irão depender do escopo das questões apresentadas. A descrição do contexto precisa ser suficientemente detalhada de modo que aqueles aspectos que possam ter uma influência significativa sobre a usabilidade possam ser reproduzidos;

- Valores reais ou desejados de eficácia, eficiência e satisfação para os contextos pretendidos. [...] (ASSOCIAÇÃO..., 2002, p. 4).

Com base na definição proposta pela NBR 9241-11, foi adotado o termo usabilidade e o método descrito nesta norma, uma vez que a mesma caracteriza de forma satisfatória a avaliação pretendida nesta pesquisa. Contudo, faz-se necessário fixar detalhadamente os indicadores a serem utilizados para medir os níveis de eficácia, eficiência e satisfação dos usuários, adaptando-os aos recursos disponíveis no Portal e aos hábitos e padrões de uso dos pesquisadores a serem estudados. Definimos assim, como indicadores para medir a usabilidade: o desempenho (eficácia e eficiência), em relação aos objetivos do produto; a satisfação dos usuários, entendida como a ausência do desconforto e presença de atitudes positivas para com o uso do produto; e o contexto de uso, ou seja, usuários, tarefas, equipamentos, e ambiente físico e social no qual o produto é utilizado.

A legibilidade possui varias implicações, por abarcar um conjunto de investigações que vão desde a perspectiva de seus caracteres, até a facilidade e rapidez da leitura dos textos. Assim, não se pode dar uma definição precisa com relação ao método mais adequado a ser utilizado para a análise da legibilidade. Para Gruszynski (2000, p.30) "entende-se pelo termo as qualidades [...] que possibilitam ao leitor reconhecer e compreender as formas e o arranjo dos tipos com maior facilidade".

No entanto, para legibilidade existem signos essenciais de textos e figuras, espaçamento dos parágrafos, entre linhas, pontuação, entre outros, que, embora sendo exigências comuns para a boa legibilidade de qualquer registro, variam de acordo com a particularidade de cada recurso. Por tal razão, cada condição de leitura de textos e objetos está estreitamente ligada a características próprias, de acordo com o contexto no qual estão inseridos e por meio dos mecanismos utilizados em sua disponibilização 
A interface é fator determinante para uma boa legibilidade do registro, pois sua formatação interfere diretamente na sua visualização, para a qual o conjunto e a sintonia das cores, fontes e do modo como as informações estão dispostas, são fatores fundamentais para haver linearidade do conteúdo disponibilizado. Dessa forma, barreiras no processo de comunicação e de transmissão da mensagem são eliminadas, facilitando assim o acesso e o entendimento.

Assim, a partir das observações e posterior mensuração dos dados obtidos com instrumento de coleta de dados, optou-se por fazer uma análise superficial da interface do Portal, ou seja, do seu layout, identificando os aspectos mais relevantes a partir das observações mais comuns entre os respondentes, para poder medir a sua legibilidade. Todavia, foi necessário utilizar os indicadores que mais se adaptaram à realidade digital, que tem como elemento fundamental a sua interface, como o excesso de informações nas telas; disposição dos menus; linearidade das informações disponibilizadas e formatação confortável para a leitura das telas (signos essenciais de textos e figuras, cores, espaçamento dos parágrafos, entre linhas, pontuação entre outros).

\section{Método}

Para alcançar o objetivo delineado nesse estudo optou-se por uma análise exploratória, uma vez que os estudos já realizados, ainda que em número reduzido, não escolheram com precisão os indicadores utilizados na avaliação do Portal de Periódicos da Capes. Julgou-se assim este método como sendo o mais adequado.

Inicialmente foi realizado um levantamento dos pesquisadores das áreas de Comunicação e Ciência da Informação da Universidade Federal da Bahia (UFBA), do qual foram selecionados todos os docentes efetivos da Faculdade de Comunicação (FACOM) e do Instituto de Ciência da Informação (ICI) da UFBA. Após coletar os nomes dos docentes nos sites das respectivas unidades, conferiu-se os nomes através de um check list fornecido pelos departamentos, sendo eliminados os docentes de contrato temporário e os que estavam afastados da atividade de ensino, totalizando 51 docentes, sendo 33 da FACOM e 18 do ICI.

Como instrumento de coleta de dados foi utilizado um questionário direcionado aos sujeitos da pesquisa a fim de verificar o grau de satisfação com a interface do Portal - identificando suas preferências quanto à organização da informação eletrônica - e testar suas habilidades no manejo do Portal. Com o término da aplicação dos questionários foi confeccionado um banco de dados utilizando o Excel (2003) para melhor tabular, sistematizar e analisar os dados.

\section{Análise e discussão dos dados}

A população investigada abrangeu um total de 51 docentes nas áreas de Comunicação e Ciência da Informação da Universidade Federal da Bahia. Destes, responderam 23 pesquisadores, sendo $11(48 \%)$ da 
Faculdade de Comunicação e 12 (52\%) do Instituto de Ciência da Informação.

Analisando o aspecto de consulta aos materiais informacionais disponibilizados pelo Portal, um dos itens analisados foi o idioma de maior acesso pelos sujeitos da pesquisa. A língua inglesa apresentou uma freqüência relativa de $50 \%$ da preferência de acesso, sendo o espanhol o segundo idioma de maior preferência pelos pesquisadores, obtendo $30 \%$ do total geral, seguido pelo francês (24\%) e outros idiomas $(9 \%)$. Constatando assim o inglês como o idioma com maior freqüência de acesso, vale destacar a hegemonia da indústria da informação, sobretudo nos EUA, uma vez que as publicações disponibilizadas no Portal são provenientes dos Estados Unidos da América e da Inglaterra, predominando assim o inglês.

$O$ resultado obtido confirma que o inglês continua sendo o idioma mais utilizado, dado já encontrado anteriormente em uma pesquisa sobre a política brasileira de publicações científicas realizada por Schwartzman (1984).

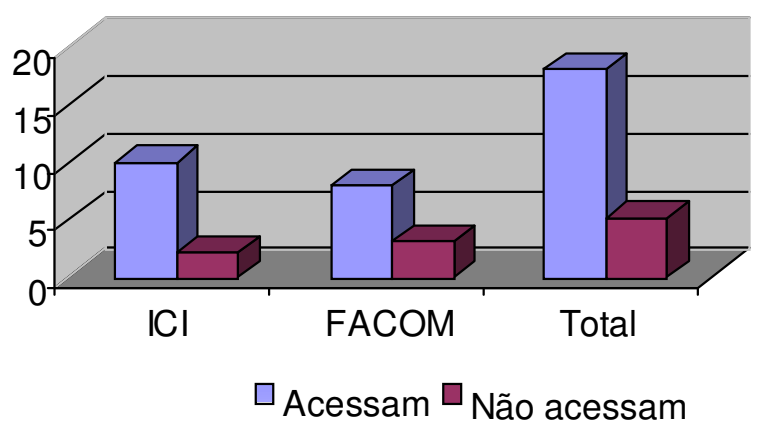

$84 \%$

$16 \%$

GRÁFICO 1 - Números de acesso ao Portal de Periódicos da CAPES.

FONTE - Dados da pesquisa.

O GRAF. 1 retrata que no quesito "acesso ao Portal", da amostra de 23 pesquisadores, $84 \%$ acessam o Portal e apenas três, o que corresponde a $16 \%$, não acessa. Contudo, é valido ressaltar que, do percentual que não acessa o Portal, $100 \%$ afirmaram saber da sua existência e ressaltaram a sua importância. Resultados que vêm corroborar o elevado número de acessos apontado em uma pesquisa realizada pelo próprio Portal CAPES (2005), onde a UFBA, com 136.355 acessos, se destacou entre as primeiras instituições do Norte-Nordeste.

\subsection{Avaliação da usabilidade e da legibilidade}

Para atender à proposta de avaliar a usabilidade e legibilidade do Portal, foi necessário mapear primeiramente o contexto de uso no quais os usuários estão inseridos, pois tal indicador será fator preponderante para mensurar os outros indicadores utilizados na avaliação da usabilidade e legibilidade. 
A fim de verificar o tipo de conexão utilizada, os dados indicam que $95 \%$ dos pesquisadores utilizam a banda larga para acessar o Portal, o que permite uma maior velocidade na taxa de transferência de dados. Verificou-se também que $85 \%$ dos usuários acessam o Portal do seu próprio local de trabalho, no caso, da universidade, que possui, nos equipamentos das suas respectivas unidades, acesso banda larga satisfatório para o uso do Portal. Esses dados nos levam a constatar que os respondentes consideram o contexto de uso apto para proporcionar o manuseio dos recursos disponibilizados pelo Portal, sem que o mesmo venha a influenciar no seu desempenho enquanto recurso eletrônico. Desse modo, consideramos que o contexto de uso é comum à maioria dos pesquisadores.

\subsubsection{Avaliação da usabilidade}

A partir dos indicadores propostos para medir a usabilidade, e com base na definição proposta pela NBR 9241-11, já descritos no item 1 do presente artigo, analisaremos o seu desempenho. As medidas de desempenho estão relacionadas com o nível de eficiência e de eficácia do uso do produto. Para mensurar o seu desempenho foram avaliados alguns recursos fundamentais destacados pelos respondentes, que são: as relações de entrada e saída de dados, utilizando como parâmetro a velocidade de abertura das telas e a eficiência dos mecanismos de busca.

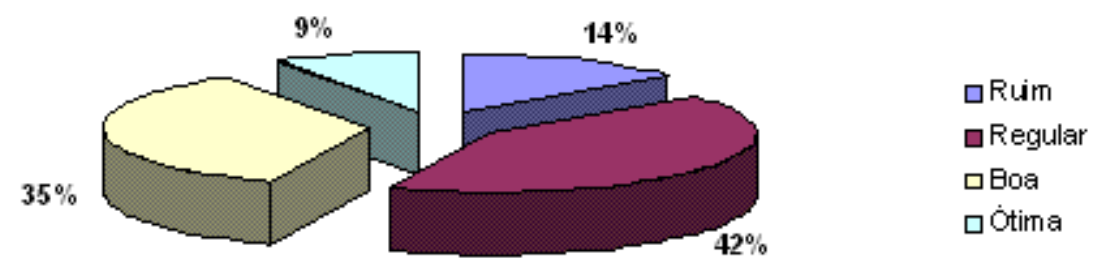

GRÁFICO 2 - Avaliação da Usabilidade

FONTE - Dados da pesquisa.

No GRÁF. 2 são apresentados os parâmetros utilizados para avaliar o desempenho, ou seja, a velocidade de abertura das telas e a eficiência na busca. Dos 23 sujeitos da pesquisa, $44 \%$ consideraram os parâmetros utilizados para a avaliação entre bom e ótimo, $42 \%$ consideraram razoável e $14 \%$ ruim, o que indica um bom desempenho do sistema na entrada e saída de dados.

Os dados analisados na avaliação do desempenho condizem com a opinião dos respondentes sobre o desempenho de todos os recursos 
oferecidos pelo Portal, visto que $49 \%$ o consideraram entre bom e ótimo, $42 \%$ regular e $9 \%$ ruim. Consideram, assim, que o Portal possui um desempenho satisfatório.

\subsubsection{Avaliação da legibilidade}

Havendo várias implicações estreitamente ligadas ao seu contexto de uso e à habilidade no uso dos recursos do Portal por parte dos usuários, optou-se por fazer uma análise superficial da sua interface, ou seja, do seu layout, identificando os aspectos mais relevantes, a partir das observações mais comuns entre os respondentes, para poder medir a sua legibilidade.

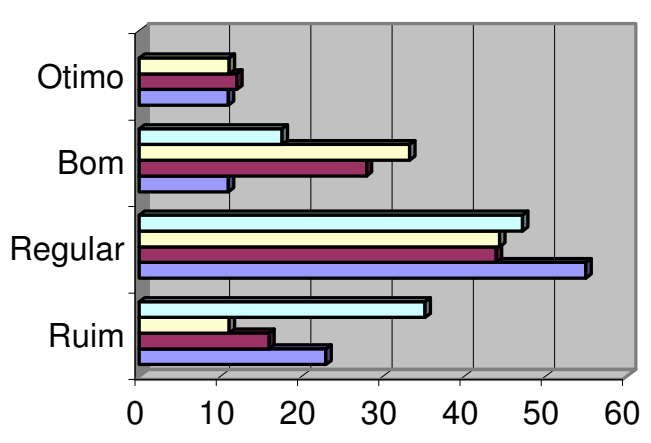

$\square$ Excesso de
informações nas
telas
$\square$ Disposição dos
menus
$\square$ Lineridade das
informações
disponibilizadas
$\square$ Telas confortaveis
para leitura

GRÁFICO 3 - Avaliação da legibilidade.

FONTE - Dados da pesquisa.

A análise aponta para a necessidade de mudanças na estrutura atual do layout apresentado no Portal (GRÁF. 3). Assim, $46 \%$ dos sujeitos consideraram esse quesito regular e $20 \%$ consideraram o layout como ruim. Para esse grupo de usuários, os indicadores levantados não são suficientemente adequados para uma boa legibilidade. Entretanto, $21 \%$ os consideraram como bons e $10 \%$ como ótimos.

O excesso de informação na tela é o maior fator de insatisfação por parte dos sujeitos dessa pesquisa, uma vez que a quantidade de informações dispostas no layout prejudica a navegação dentro do sistema. Analisando esse dado juntamente com os dados apresentados no GRAF. 2, a avaliação da legibilidade reforça a necessidade de melhorias na interface do Portal.

\section{Conclusões e recomendações}

Para se alcançar o objetivo delineado nesse estudo, que foi o de investigar um grupo selecionado de pesquisadores das áreas de Comunicação e Ciência da Informação/UFBA, para verificar o grau de aceitação e satisfação que eles demonstram em relação à funcionalidade e 
ao desempenho do Portal de Periódicos da Capes, optou-se por privilegiar o diagnóstico da interface eletrônica do Portal, avaliando a sua usabilidade e legibilidade, chegando aos resultados descritos abaixo.

\subsection{Usabilidade}

A partir dos indicadores propostos para medir a usabilidade, observa-se o desempenho (eficácia e eficiência) em relação aos objetivos do produto e a satisfação dos usuários, entendida como ausência de desconforto e presença de atitudes positivas para com o uso do produto:

- $84 \%$ do total dos respondentes acessam o Portal e apenas $16 \%$ não acessam;

- Os respondentes indicam que o sistema possui um bom desempenho na entrada e saída de dados, sendo que $60 \%$ deles apontaram como bom e ótimo. Isso reforça a opinião dos respondentes em relação ao desempenho de todos os recursos oferecidos pelo Portal: $42 \%$ consideraram regular, $49 \%$ entre bom e ótimo e apenas $9 \%$ ruim, tendo-se assim um grau relevante de satisfação na opinião dos sujeitos dessa pesquisa.

\subsection{Legibilidade}

Em relação à análise das variáveis propostas na avaliação da legibilidade, a maior parcela dos respondentes (46\%) considerou o layout regular e $20 \%$ ruim. Para esse grupo de usuários, os indicadores levantados não são suficientemente adequados para uma boa legibilidade. Entretanto, $21 \%$ a consideraram como boa e $10 \%$ como ótima.

Os resultados da pesquisa sugerem que a avaliação adotada foi satisfatória, pois o Portal apresentou, de uma forma geral, um bom nível de usabilidade e legibilidade, de acordo com os dados coletados, necessitando apenas de algumas melhorias para um desempenho mais eficiente. Quanto ao aprimoramento da usabilidade e da legibilidade do Portal de Periódico da CAPES, seguem-se algumas recomendações baseadas nas análises realizadas:

- o sistema é pouco interativo, tanto na entrada como na saída dos dados, especialmente em relação ao refinamento da busca, o que sugere uma reformulação na metodologia utilizada;

- a reformulação do layout contribuiria para a melhoria da legibilidade e da usabilidade do sistema, especificamente com a retirada do excesso de informações nas telas do Portal, principalmente na tela principal, objeto da maior insatisfação dos usuários, e que prejudica de forma significativa a leitura e a recuperação de informações dentro do Portal;

- sugere-se a implantação de um serviço de alerta bibliográfico, já que $100 \%$ dos respondentes se mostraram insatisfeitos com a falta de informações em relação aos títulos disponíveis e às novas aquisições;

- Assegurar maior esclarecimento e ampliação da FAQ e da ajuda; 
- Possibilitar maior transparência na aquisição e nos parâmetros utilizados nas aquisições do Portal, além de criar mecanismos mais interativos com a participação mais efetiva dos usuários na seleção dos títulos disponíveis no Portal.

Espera-se que a pesquisa realizada contribua para avaliar a eficiência do sistema e para permitir melhorias na sua funcionalidade e desempenho. Contudo, é valido ressaltar que as terminologias e metodologias mencionadas ainda serão aprimoradas em estudos posteriores.

\section{REFERÊNCIAS}

ASSOCIAÇÃO BRASILEIRA DE NORMAS TÉCNICAS. NBR 9241-11 Requisitos ergonômicos para trabalho de escritórios com computadores. Parte 11 - Orientações sobre usabilidade. Rio de Janeiro: ABNT, 2002.

COORDENAÇÃO DE APERFEIÇOAMENTO DE PESSOAL DE NÍVEL SUPERIOR - CAPES. Portal de Periódicos. Disponível em: <www.periodicos.capes.gov.br>. Acesso em: 15 dez. 2005.

GRUSZYNSKI, A. C. Design gráfico: do invisível ao ilegível. Rio de Janeiro: $2 A B, 2000$.

MEIRELLES, R. F. A funcionalidade e o desempenho do Portal de Periódicos da CAPES entre pesquisadores das áreas de Comunicação e Ciência da Informação da Universidade Federal da Bahia. 39 f. 2005. Monografia (Graduação em Biblioteconomia e Documentação) - Instituto de Ciência da Informação da Universidade Federal da Bahia, Salvador.

SARACEVIC, T.; COVI, L. Challenges for digital library evaluation. Paper submitted to the 2000 Annual Meeting of the American Society for Information Scence. 13p. Disponivel em: $<$ www. is.informatik. uniduisburg.de/courses/dl ss04/folien/saracevic0 0.pdf >. Acesso em: 25 set. 2005.

SCHWARTZMAN, S. Política brasileira de publicações científicas e técnicas: reflexões. Revista Brasileira de Tecnologia, Brasília, v.15, n.3, maio/jun, 1984. 